\title{
Antibiotic-mediated changes in the fecal microbiome of broiler chickens define the incidence of antibiotic resistance genes
}

Wenguang Xiong ${ }^{1,2}$, Yulin Wang ${ }^{2}$, Yongxue Sun ${ }^{1}$, Liping $\mathrm{Ma}^{2}$, Qinglin Zeng ${ }^{1}$, Xiaotao Jiang ${ }^{2}$, Andong Li ${ }^{2}$, Zhenling Zeng ${ }^{1 *}$ and Tong Zhang ${ }^{2^{*}}$ (D)

\begin{abstract}
Background: Antimicrobial agents have been widely used in animal farms to prevent and treat animal diseases and to promote growth. Antimicrobial agents may change the bacterial community and enhance the resistome in animal feces. We used metagenome-wide analysis to investigate the changes in bacterial community, variations in antibiotic resistance genes (ARGs), and their bacterial hosts in the feces of broiler chickens over a full-treatment course of chlortetracycline at low and therapeutic dose levels.

Results: The effects of chlortetracycline on resistome were dependent on the specific ARG subtypes and not simply the overall community-level ARGs. Therapeutic dose of chlortetracycline promoted the abundance of tetracycline resistance genes (tetA and tet $\mathrm{W}$ ) and inhibited multidrug resistance genes ( $m d t \mathrm{~A}, m d t C, m d t \mathrm{~K}, o m p R$, and To/C). The therapeutic dose of chlortetracycline led to loss of Proteobacteria mainly due to the decrease of Escherichia/Shigella (from 72 to 58\%). Inhibition of Escherichia by chlortetracycline was the primary reason for the decrease of genes resistant to multiple drugs in the therapeutic dose group. The ARG host Bifidobacterium were enriched due to tetW harbored by Bifidobacterium under chlortetracycline treatment. Escherichia was always the major host for multidrug resistance genes, whereas the primary host was changed from Escherichia to Klebsiella for aminoglycoside resistance genes with the treatment of therapeutic dose of chlortetracycline.

Conclusions: We provided the first metagenomic insights into antibiotic-mediated alteration of ARG-harboring bacterial hosts at community-wide level in chicken feces. These results indicated that the changes in the structure of antibiotic-induced feces microbial communities accompany changes in the abundance of bacterial hosts carrying specific ARGs in the feces microbiota. These findings will help to optimize therapeutic schemes for the effective treatment of antibiotic resistant pathogens in poultry farms.
\end{abstract}

Keywords: Metagenome, Antibiotic resistance genes, Resistome, Bacterial community, Feces, Chickens

\section{Background}

Antibiotic resistance is one of the most serious global threats to human health, as strongly evidenced by the serious implications of the recent worldwide emergence of KPC-2 (Klebsiella pneumoniae carbapenemase-2), NDM-1 (New Delhi metallo- $\beta$-lactamase-1), and MCR-1

\footnotetext{
* Correspondence: zlzeng@scau.edu.cn; zhangt@hku.hk

${ }^{1}$ National Laboratory of Safety Evaluation (Environmental Assessment) of Veterinary Drugs (SCAU) and Guangdong Provincial Key Laboratory of Veterinary Pharmaceutics Development and Safety Evaluation, South China Agricultural University, 483 Wushan Road, Guangzhou 510642, China 2Environmental Biotechnology Laboratory, The University of Hong Kong, Pokfulam Road, Hong Kong, China
}

(plasmid-mediated colistin resistance) [1-4]. Animal fecal microflora harbors a vast reservoir of antibiotic resistance genes (ARGs) that could be acquired by human commensals and pathogens [5]. The antibiotic residues, resistant bacteria, and ARGs in animal excretion may be transported into the environment via manure application, leakage, runoff, and airborne particulate matter [6-8], globally contributing to the aggregation of resistance in the environment.

Poultry production is one of the fastest growing industries worldwide. The global population will reach 8.5 billion by 2030 [9], posing high pressure on international 
poultry production including the world's largest poultry producer, China [10]. Large-scale intensive farming systems depend on antimicrobials to prevent and treat animal disease and to enhance growth performance. It is estimated that the global consumption of antimicrobials used for chickens, pigs, and cattle will increase by $67 \%$, from 63,151 tons in 2010 to 105,596 tons in 2030 [11].

Much of the current data regarding the gastrointestinal microbiome in poultry is centered on bacterial communities associated with poultry growth performance and disease status, thus having implications for human health. For examples, Oakley et al. (2014) investigated the effects of formic acid, propionic acid, and medium-chain fatty acids as feed additives on pathogens and found that these treatments did not affect specific pathogens or the cecal microbiome composition [12]. Costa et al. (2017) investigated the impact of antibiotic growth promoters such as zinc bacitracin, enramycin, halquinol, virginiamycin, and avilamycin on the cecal microbiota of broiler chicken and found specific changes in cecal microbiota membership regarding antibiotic growth performance [13]. Wei et al. (2013) examined the effects of supplemental bacitracin and litter management on food-borne enteric pathogens in broiler chickens [14].

Antibiotic administration has profound effects on indigenous microbes of animal feces, leading to changes in microbial community structure and resistance. Chlortetracycline is one of the most commonly used antimicrobials in poultry and swine production [15]. China is the world's largest producer and consumer of chlortetracycline for veterinary use [16]. However, the changes in microbial community and bacterial resistome in the poultry gastrointestinal tract and resultant feces following chlortetracycline treatment remain largely unknown.

Recently, phages were shown to contribute to antibiotic resistance [17], but they rarely encoded ARGs [18]. Horizontal gene transfer (HGT) via mobile genetic elements such as plasmids, integrases, and transposases are important drivers of ARG dissemination. However, the bacterial community structure rather than HGT has been observed to be the primary determinant of the ARG content in soils [19]. Bacterial community shifts were strongly correlated with the occurrence of antibiotic resistance alterations during drinking water chlorination [20]. The relationship between ARGs and bacterial communities has not been characterized in the feces of animals such as poultry. The identification of bacterial hosts harboring ARGs in poultry feces is helpful for us to optimize a therapeutic scheme to avoid the spread and aggregation of antibiotic resistance.

ARGs can be transported into the environment and acquired by human microbiota via poultry feces used as organic fertilizers for vegetables and fruits [21]. A deep quantitative understanding of the distribution and diversity of ARGs and their bacterial hosts in poultry feces is necessary to evaluate their co-occurrence while they are discharged into the environment and acquired by human pathogens. ARG abundance and diversity were underestimated by previous studies based on cultured bacteria and identified ARGs [22], which is emphasized by increase of sequence-novel ARGs [23]. Sequence-based metagenomic approaches allow a comprehensive exploration of uncultivable and rare taxa from the immense diversity in the fecal microbial population. Recently, although metagenomic approaches have been used to analyze the bacterial communities [24, 25] and ARG abundance in poultry feces [26-28], the responses of ARG-harboring bacterial hosts to antibiotic treatments have been neglected. Chlortetracycline is one of the most commonly used antimicrobials in poultry production for disease prevention and treatment. These diseases include infectious synovitis and air sacculitis that are treated for 3 to 5 days as one course of treatment. Chlortetracycline is also routinely and extensively used in feed for weight gain/feed efficiency in the absence of disease signs and symptoms [29].

We hypothesize that the changes in the structure of antibiotic-induced fecal microbial communities accompany changes in the abundance of bacterial hosts carrying specific ARGs in chicken fecal microbiota. To test this hypothesis, we investigated the impact of a 5-day full-treatment course of chlortetracycline administration on the fecal resistome and microbiota of chickens. By applying 16S rRNA amplicon sequencing and de novo assembly of metagenomic data set, we profiled the changes in fecal bacterial community, the variations in ARG, and bacterial host abundance to demonstrate that antibiotic-induced changes in the abundance of bacterial hosts shape ARG abundance in chicken feces.

\section{Methods \\ Chicken and chlortetracycline exposure}

Fifty-four 20-day-old chickens were purchased in the same batch from a commercial production (Guangdong Wens Dahuanong Biotechnology Co., Ltd., China). From days 15 to 22, microbiota maturation occurs and remains in a stable status [25]. We therefore chose 20-day-old chickens for our experimental procedures. At the commercial production, these chickens were raised in cages under standard commercial conditions and had not been treated by any antimicrobials. The chickens were transported to Laboratory Animal Center of South China Agricultural University and divided into three groups (see Additional file 1: Figure S1). Each group had three replicates. Each replicate had six chickens per cage with equal representations of body weight and sex. The chickens were raised in cages and fed under standard commercial production conditions and received a 
standard commercial corn-soy diet (Yellow little chicken feed, Guangdong Wens Dahuanong Biotechnology Co., Ltd., China) and water ad libitum. The chickens were raised for 7 days before chlortetracycline administration. A 5-day course of chlortetracycline was administered at $2 \mathrm{~g} / \mathrm{L}$ (the therapeutic dose group) and $0.2 \mathrm{~g} / \mathrm{L}$ (the lowdose group) in the drinking water. One group without chlortetracycline administration was set as the control group. Each group had three cages, and each cage contained six chickens. Feces from every cage were cleaned daily. Mixed fresh feces from six chickens in each cage were collected on T0 (day 0 , before treatment), T5 (day 5, during treatment), and ST10 and ST20 (day 10 and 20, respectively, during stopped treatment) (see Additional file 1: Figure S1). The fresh feces were collected into sterilized tubes using a sterilized spoon, and the sterilized tube was put into an aseptic plastic bag. Three biological replicates were conducted in the treatment and control groups. All the samples were kept on ice and were immediately transferred to laboratory for processing. Chlortetracycline administration was performed from 8 August, 2015, to 28 August, 2015. No clinical symptoms or signs such as diarrhea, allergy, and other disease outbreaks were observed in these chickens during the experiment.

\section{DNA extraction}

Fresh feces from six chickens in one cage were mixed as one replicate. DNA was extracted from three biological replicates using MoBio PowerSoil DNA isolation kit (MoBio Laboratories, Carlsbad, CA, USA) according to the Human Microbiome Project protocol. DNA concentrations and purity were measured by UV spectroscopy using a NanoDrop ND-2000 instrument (NanoDrop Technologies, Wilmington, DE, USA).

\section{S rRNA gene profile}

Sequencing of $16 \mathrm{~S}$ rRNA gene was performed using Illumina MiSeq with a paired-end 300-bp sequencing strategy. The $16 \mathrm{~S}$ primers ( $319 \mathrm{~F}$ and $806 \mathrm{R}$ ) were used to amplify the hypervariable V3-V4 region of bacterial $16 \mathrm{~S}$ rRNA genes [30]. Primers also included Illumina sequencing adapter, pad, linker, and the reverse primer contained a 12-bp error-correcting barcode [31]. PCR was performed using a Bio-Rad thermal cycler Model C1000 (Bio-Rad, Richmond, CA, USA). The reaction mixture $(50 \mu \mathrm{L})$ consisted of $1 \mu \mathrm{L}$ of DNA template, $0.5 \mu \mathrm{L}$ of each primer $(50 \mathrm{pmol}), 5 \mu \mathrm{L}$ of $10 \times E x$ Taq buffer containing $20 \mathrm{mM} \mathrm{Mg}{ }^{2+}, 0.25 \mu \mathrm{L}$ of $E x$ Taq DNA polymerase, and $5 \mu \mathrm{L}$ of dNTP mixture $(2.5 \mathrm{mM})$. The PCR procedure was as follows: initial $94{ }^{\circ} \mathrm{C}$ denaturation for $3 \mathrm{~min}$, followed by 30 cycles consisting of denaturation $\left(94{ }^{\circ} \mathrm{C}\right.$ for $\left.30 \mathrm{~s}\right)$, annealing $\left(50{ }^{\circ} \mathrm{C}\right.$ for $\left.30 \mathrm{~s}\right)$, extension $\left(72{ }^{\circ} \mathrm{C}\right.$ for $\left.30 \mathrm{~s}\right)$, and a final extension step $\left(72{ }^{\circ} \mathrm{C}\right.$ for $10 \mathrm{~min})$. No-template controls were included as negative controls. Clean reads were obtained using the mother MiSeq standard operating procedure (http:// www.mothur.org/wiki/MiSeq_SOP) [31]. Briefly, $16 \mathrm{~S}$ rRNA gene reads were denoised by using pre.cluster, and chimeric sequences were checked by Uchime [31]. On average, we obtained 33,123 clean reads for each sample (Additional file 2: Table S1). We then picked operational taxonomic units (OTU) at 97\% sequence identity using the QIIME analysis pipeline [32]. Taxonomic assignments were classified at $80 \%$ bootstrap confidence using the Ribosome Database Project [33]. For comparisons across groups, the sequencing depth of samples was normalized to 18,888 reads.

\section{Metagenomic sequencing}

The DNA from fecal samples for $16 \mathrm{~S}$ rRNA gene sequencing was simultaneously used for metagenomic sequencing. Metagenomic sequencing was performed using Illumina Hiseq 4000 with the sequencing strategy of Index $150 \mathrm{PE}$ (paired-end sequencing). Low-quality reads were filtered to ensure that (1) the reads were aligned to proper adaptors or primers, (2) the reads contained $<10 \%$ unknown bases, and (3) the reads contained $>50 \%$ high-quality bases [34]. On average, $8.7 \mathrm{~Gb}$ clean reads were generated for each sample.

\section{Calculations of ARG abundance}

ARG abundance was determined using ARGs-OAP [35]. Briefly, potential ARG reads and 16S rRNA genes were extracted, and ARG-like reads were identified and annotated using BLASTX by applying the combined ARG database of CARD (The Comprehensive Antibiotic Resistance Database) and ResFinder (freely accessed on 8 September, 2017). Reads were annotated as ARG-like reads at the cutoff of $E$ value of $10^{-7}$, sequence identity of $90 \%$ and alignment length more than 25 amino acids. This identification approach had been validated to $99.5 \%$ accuracy by our group $[28,36]$. The abundance of ARGlike reads was calculated and normalized by the number of 16S rRNA genes (defined as relative abundance). ARG abundance was expressed as copies of ARGs per copy of $16 \mathrm{~S}$ rRNA gene in order to be directly compared with the results acquired using qPCR [28]. ARG types and subtypes were automatically counted by using a package of customized scripts previously described by our group [36].

\section{Metagenome assembly}

Clean reads in three replicates for each treatment were de novo assembled with default $k$-mer size using the CLC Genomics Workbench (Version 6.0.2, CLC Bio, Aarhus, Denmark). We obtained 148,151 contigs with average length of $1690 \mathrm{bp}$ (Additional file 2: Table S2). 


\section{Identification of ARG-like ORFs}

Open reading frames (ORFs) within contigs were predicted using Prodigal v.2.60 [37]. ORF coverage was calculated by mapping metagenomic reads to the contigs with a minimum length coverage of 95 at $95 \%$ similarity [27]. ARG-like ORFs were determined using BLASTX against the combined ARG database mentioned above at $E$ value $\leq 10^{-10}$ [38] with a minimum similarity of $80 \%$ over $70 \%$ of query coverage [27]. Coverage (times per Giga base, $\times / G b)$ of ARG-like ORFs were defined as follows:

$$
\text { Coverage }=\sum_{1}^{n} \frac{N \times 150 / L}{S}
$$

where $N$ is the number of reads mapped to ARG-like ORFs, $L$ is the sequence length of target ARG-like ORFs, $n$ is the number of ARG-like ORFs, 150 is the length of Illumina sequencing reads, and $S$ is the sequencing data size $(\mathrm{Gb})[27]$.

\section{Taxonomic assignment of bacterial hosts of ARGs}

Protein sequences of ORFs predicted in the contigs that carried ARG-like ORFs were annotated using BLASTP against NCBI NR database (downloaded on 2 February, $2015)$ at $E$ value $\leq 10^{-5}$. The BLASTP results were annotated using MEGAN (MEtaGenome ANalyzer, Version 5) [39] for assignment of taxonomic genus. The contig was assigned to the taxon if the voting score was more than $50 \%[11,40]$.

\section{Statistical analysis}

Statistical comparisons were done using nonparametric Kruskal-Wallis tests. A $P$ value of $<0.05$ was regarded as statistically significant. Hierarchical clustering was conducted using the similarity index of Bray-Curtis by the algorithm of unweighted pair-group method with arithmetic averages.

\section{Submission of sequence data}

All sequence data have been deposited in the NCBI Sequence Read Archive (accession no. SRP091637 for metagenomic analysis and accession no. SRP091694 for $16 \mathrm{~S}$ analysis).

\section{Results}

\section{ARG variations}

On average, we found 5.1 ARG copies per 16S rRNA gene calculated from all fecal metagenomes of broiler chickens (Additional file 2: Table S3). The predominant ARG types were multidrug resistance genes (46\%), aminoglycoside resistance genes (10\%), and tetracycline resistance genes (6.5\%) (Additional file 1: Figure S2). Metagenomic analysis showed that the ARGs were diverse and abundant in feces of broiler chickens, even when chlortetracycline was not administered in control animals. In these control groups, the relative abundances of total ARGs ranged from 4.3 to 6.0. Chlortetracycline treatment at low or therapeutic doses did not significantly alter the relative abundance of total ARGs, nor did it result in increases in the three predominant ARG types (Additional file 1: Figure S2).

We also found that genes resistant to multidrugs and tetracyclines occurred predominantly via drug transporters (Additional file 2: Table S4). For example, the multidrug resistance genes $m d t E, m d t \mathrm{~L}, m d t \mathrm{P}, m d t \mathrm{~N}$, and $m d t \mathrm{~F}$ were in the 20 most abundant ARG subtypes. Interestingly, tet $\mathrm{A}$ was the most abundant ARG subtype. In contrast, most aminoglycoside and $\beta$-lactam resistance genes uncovered ARGs with antibiotic-inactivating capabilities, via covalent modification of aminoglycosides ( $a a d \mathrm{~A}$ and $a a d \mathrm{E})$ and enzymatic degradation of $\beta$ lactams (class $C \beta$-lactamase). The quinolone resistance gene $q n r S$ was primarily plasmid associated. Importantly, mcr-1 gene occurred as high frequencies. This gene was first reported by our laboratory as a plasmid-mediated polymyxin resistance gene [3]. We located the $m c r-1$ gene on a novel contig (Additional file 1: Figure S3).

We further analyzed the ARG subtype variations to quantitatively compare the effects of chlortetracycline on the fecal bacterial resistome. The shift of samples as illustrated by principal component analysis (PCA) suggested an impact of chlortetracycline on the fecal resistome at the level of ARG subtypes over time (Fig. 1a). The tetracycline resistance genes (tet $\mathrm{A}$ and tet $\mathrm{W})$ significantly increased in dosedependent manner in the low-dose and therapeutic dose treatment groups on T5 $(P<0.05)$. In contrast, some ARG subtypes significantly decreased due to chlortetracycline treatment (on T5), such as several multidrug resistance genes $(m d t \mathrm{~A}, m d t \mathrm{C}, m d t \mathrm{~K}, o m p \mathrm{R}$, and $T o l \mathrm{C})$ (Fig. $1 \mathrm{~b}$ and Additional file 2: Table S4).

\section{Changes in bacterial community structure}

We analyzed bacterial community structure using sequence data of $16 \mathrm{~S}$ rRNA genes. We found that Proteobacteria (81\%), Firmicutes (11\%), Bacteroidetes (2.7\%), and Actinobacteria (1.3\%) were the predominant taxonomic phyla in fecal microbiota of broiler chickens (Additional file 1: Figure S4 and Additional file 2: Table S5). These four main taxonomic phyla also represented the predominant human fecal microbiota [41]. Our data contrasts with a previous study that found Firmicutes (56\%), Bacteroidetes (36\%), and Proteobacteria (1.7\%) from chicken feces using the same sequencing approach [42].

There were no significant compositional shifts of the predominant Proteobacteria and Firmicutes in the lowdose group compared with the control group over time $(n=27)$. This suggested that the structure of the fecal 

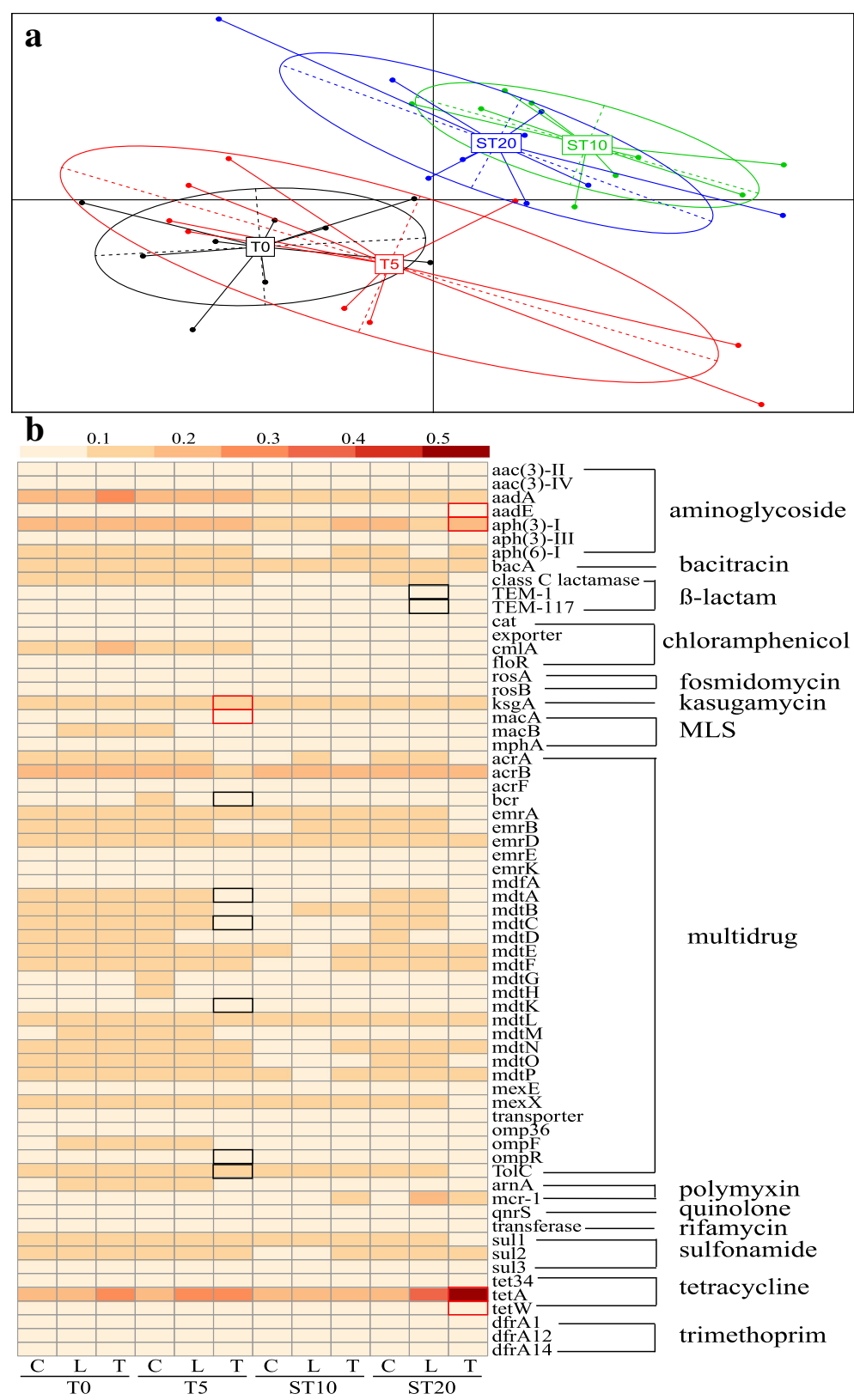

Fig. 1 Variations of ARG subtypes in different samples over the course of chlortetracycline administration. a Principal component analysis of ARG subtypes. $\mathbf{b}$ Heatmap of variations of ARG subtypes based on the relative abundance of ARG subtypes. Significant $(P<0.05)$ increases and decreases are shown by border lines of cells colored by red and black, respectively. ARG subtypes with an average abundance $>1 \%$ are shown. See Additional file 2: Table S4 for the relative abundances of ARG subtypes

taxonomic phyla was relatively stable over time, even after 5 days of low-dose chlortetracycline treatment. However, the therapeutic dose of chlortetracycline led to significant shifts in the composition of taxonomic phyla including the loss of Proteobacteria (from $83 \%$ on T0 to $75 \%$ on T5) compared with the control group $(P=0.045$, $n=27$ ) (Fig. 2a).

As the predominant genus of Proteobacteria, Escherichia/Shigella significantly decreased from $70 \%$ on T0 to
$58 \%$ on ST20 in the therapeutic dose group $(P=0.028$, $n=12$ ) (Fig. $2 \mathrm{~b}$ and Additional file 2: Table S6). In contrast, there was no significant shift in Escherichial Shigella in the control group $(P=0.16, n=12)$. The decrease of Escherichia/Shigella mediated by chlortetracycline was the major contributor to the loss of taxonomic phylum of Proteobacteria in the therapeutic dose group observed above. The overall depletion of Escherichia in the community structure corresponded to the 


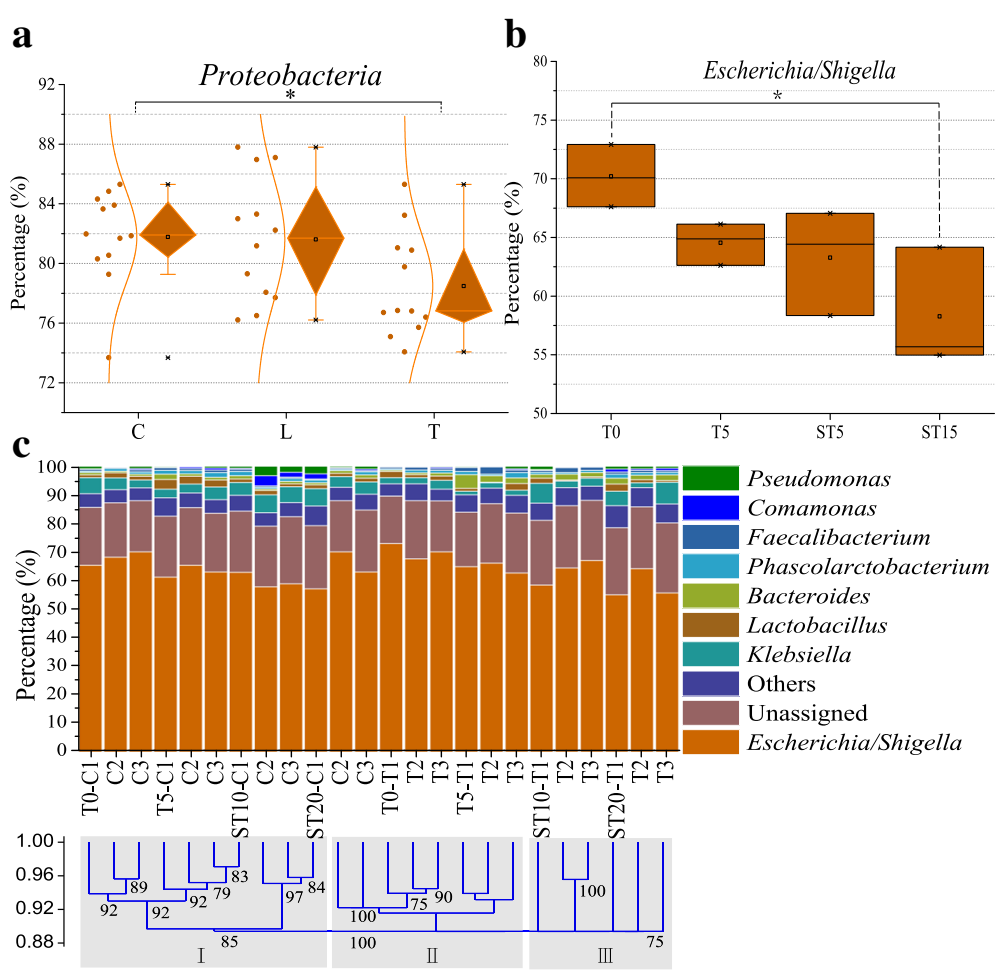

Fig. 2 Changes in bacterial community structure. a Changes in taxonomic Proteobacteria over time among the different groups. b Changes in taxonomic Escherichia/Shigella over time in the therapeutic dose group. c Changes in taxonomic genera in the therapeutic dose group compared with the control group. Others in Fig. 2c mean the percentage of each taxonomic genus $<0.5 \%$. Details of the percentage of taxonomic phyla and genera are shown in Additional file 2: Tables S5 and S6, respectively

decrease of ARG-harboring Escherichia. In addition, the therapeutic dose level of chlortetracycline shifted the fecal microbiome into a distinct structure of taxonomic genera (Fig. 2c) and was most likely due to the broadspectrum activity of chlortetracycline against both Gram-positive and Gram-negative bacteria. The taxonomic genera in the control and the therapeutic dose groups were clustered into three classes. In the control group, most samples clustered into class I. In the therapeutic dose group, the samples on $\mathrm{T} 0$ and $\mathrm{T} 5$ clustered into class II, and the samples on ST10 and ST20 clustered into class III. This indicated that the therapeutic dose of chlortetracycline resulted in the divergent changes in taxonomic genera. The divergent changes were also supported by PCA, in which PC 1 accounted for $80 \%$ of the variations between samples that were spatially separated from each group (Additional file 1: Figure S5).

\section{Changes in bacterial hosts of ARGs}

The current study achieved a greater depth of sequencing than previous studies of fecal metagenomes of poultry [27, 43], permitting an unparalleled contig assemble and comparison of ARGs. We obtained $2.08 \times$ $10^{12}$ clean reads that were assembled into $1,777,809$ contigs containing 4,105,873 ORFs with an average length of $646 \mathrm{bp}$ (Additional file 2: Table S2). We annotated 4812 ORFs as ARG-like ORFs that were located in 4443 contigs. To quantitatively compare resistomes and their bacterial hosts at higher resolution, we taxonomically assigned these ARG-carrying contigs and normalized the coverage of ARG-like ORFs.

ARGs were harbored by diverse genera including Escherichia, Klebsiella, Shigella, and Salmonella (Fig. 3a and Additional file 1: Figure S6), suggesting that the fecal resistome resided in phylogenetically diverse microbiota. Escherichia was the predominant bacterial host (83\%) and harbored most of diverse ARGs including the genes resistant to multidrug, tetracycline, aminoglycoside, macrolide lincosamide streptogramin, $\beta$-lactam, and sulfonamides. In a study of dairy cow manure, ARGs originated from Proteobacteria, Firmicutes, Bacteroidetes, and Actinobacteria [44]. More than half of genes resistant to multidrug (85\%), tetracycline (95\%), aminoglycoside $(80 \%)$, macrolide lincosamide streptogramin (76\%), and $\beta$-lactam (79\%) resided in Escherichia, whereas vancomycin resistance genes mainly distributed in Enterococcus (100\%) and fosfomycin resistance genes mainly distributed in Klebsiella (53\%) and Enterobacter (47\%). 

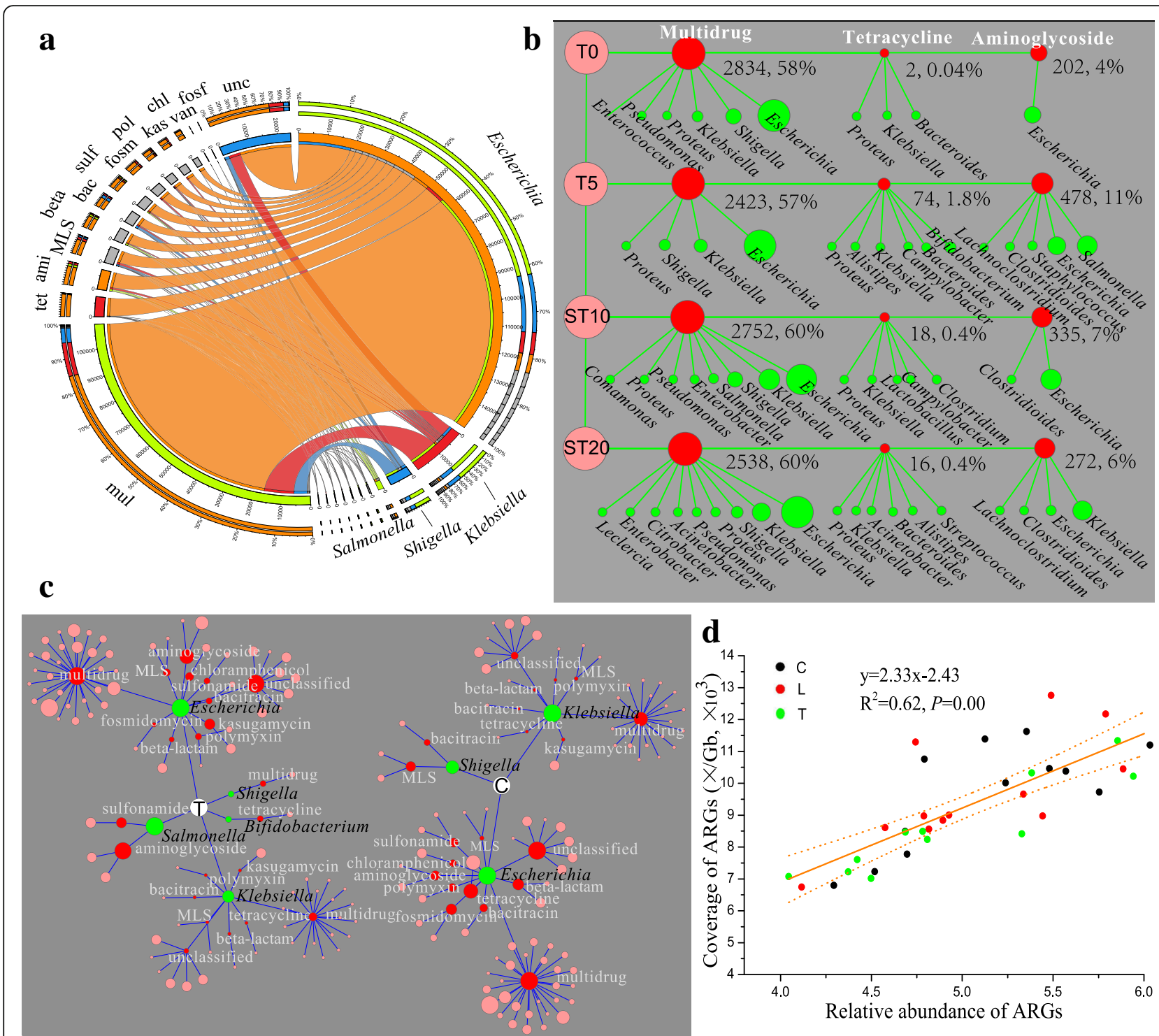

Fig. 3 ARGs and their bacterial hosts. a Percentages of ARG-carrying bacterial hosts. Mul multidrug, tet tetracycline, ami aminoglycoside, MLS macrolide-lincosamide-streptogramin, beta beta-lactam, bac bacitracin, sul sulfonamide, fosm fosmidomycin, pol polymyxin, kas kasugamycin, chl chloramphenicol, van vancomycin, fosf fosfomycin, unc unclassified. Details are shown in Additional file 1: Figure S6 and Additional file 2: Table S7. $\mathbf{b}$ Variations of the predominant ARGs carried by bacterial hosts over time in the therapeutic dose group. ARGs and bacterial hosts are colored by red and blue nodes, respectively. Node size corresponds to percentage. ARGs and their bacterial hosts are connected by line. Details are described in Additional file 2: Table S8. The percentage of a single ARG was calculated using the coverage of the ARG divided by the sum of the coverage of all ARGs in one sample. $\mathbf{c}$ Changes of bacterial hosts carrying ARGs from the control group compared to the therapeutic dose group on T5. C the control group, $T$ the therapeutic dose group. Green nodes, red nodes, and pink nodes mean hosts, ARG types, and ARG subtypes, respectively. Node size corresponds to percentage. Bacterial hosts with a percentage of $>1 \%$ are shown. Details are described in Additional file 2: Table S9. $\mathbf{d}$ Linear relationship between the relative abundance of total ARGs and the coverage of total ARGs harbored by bacterial hosts. Each dot represents one sample, and a solid line means a linear fit. The dashed lines represent $95 \%$ confidence intervals

The changes in the three most abundant ARG types significantly influenced the overall fecal bacterial resistome (Fig. 3b). During the experimental duration, Escherichia was always the major host for the multidrug resistance genes, while the major host for aminoglycoside resistance genes was Escherichia on T0, but changed to Klebsiella on ST20. The coverage of the multidrug resistance genes decreased up to $15 \%$ with antibiotic treatment on $\mathrm{T} 5$ and did not recover to the initial level on T0 at the end of the experiment on ST20 (Fig. 3b). Inhibition of Escherichia by chlortetracycline was the primary reason for the decrease of multidrug resistance genes in the therapeutic dose group. The coverage of Escherichia that harbored multidrug 
resistance genes decreased by 13 to $17 \%$ on T5, ST10, and ST20 compared with T0. This was the primary contribution to the decrease of the hosts harboring these genes (Additional file 1: Figure S7 and Additional file 2: Table S10).

The enrichment of tetracycline resistance genes was due to the emergence of a new set of bacterial hosts that included Bifidobacterium (1.3\%) on T5 (Fig. 3c). ARG hosts Bifidobacterium were enriched after chlortetracycline treatment in the therapeutic group compared to the control group. These ARG contents harbored by Bifidobacterium partially explained the enrichment of ARG-harboring hosts. We found that Bifidobacterium harbored tetW, and not surprisingly, the hosts Bifidobacterium were enriched under chlortetracycline treatment.

We further analyzed the correlation between the abundance of total ARGs (calculated by raw reads) and the coverage of total ARGs (based on metagenomic assembly) that were harbored by bacterial hosts in each sample. We found a significantly positive linear relationship $\left(y=2.33 \times-2.43, R^{2}=0.62, P=0.00\right)$, even treated by the low and therapeutic dose of chlortetracycline (red and green dots in Fig. 3d). These results clearly showed that the ARGs harbored by bacterial hosts were dictated by the total ARG reads, and it was, therefore, predictable, even with chlortetracycline treatment. The good linear relationship suggested that assembled ARGs can represent raw read ARGs, and our analysis of ARG hosts based on the contig assembly is appropriate.

\section{Discussion}

Numerous studies of antibiotic resistance using traditional bacterial culture methods have provided information concerning existing and emerging antibiotic resistance threats $[4,45]$. Over time, the study of antibiotic resistance has grown from single cultured microorganisms to exploring antibiotic resistance in diversified bacteria including pathogenic, commensal, and environmental bacteria at the level of microbial communities [46]. In the current study, we here adopted a high-throughput approach, nextgeneration sequencing, to analyze the effects of a broadspectrum antibiotic on the fecal resistome and bacterial communities in chickens, highlighting the antibioticinduced alterations of ARG-harboring bacterial hosts in microbial communities.

The most common ARG types including the genes resistant to multidrug, aminoglycoside, and tetracycline detected in this study were also found in other specific niches, such as human feces and the environment [28, 47, 48]. However, the predominant ARGs found in this study were different from soil in which $\beta$-lactamases were the most frequently encountered [19] and were also different from human feces, river water, and sediments [28]. This supported that ARGs are not randomly distributed in diverse environments. We found an average of 5 ARG copies per 16S rRNA gene in both the control and chlortetracycline treatment groups. This level of ARG abundance was greater than that observed in feces from commercial chicken flocks (3.1 copies) because of updated ARG databases [28]. The ARG levels from chickens with different backgrounds of antibiotic use indicated that the ARGs might be established in poultry fecal metagenomes in the absence of antibiotic exposure. Similarly, ARGs were established in human feces within the first week after birth independent of antibiotic treatment [49]. Regardless of antibiotic selection, early establishment of fecal bacterial resistome most likely originated from the environmental microbiota. For instance, the existence of ARGs in Hadza hunter-gatherers with little antibiotic exposure supported the ubiquitous presence of environmentally derived resistances [50], which are the weapons that environmental microorganisms use to compete against other microbes. We found that chlortetracycline treatment did not significantly increase the relative abundance of total ARGs, nor did it alter the three predominant ARG types. This suggests that the effects of antibiotics on the fecal bacterial resistome were dependent on ARG subtypes and not simply on the overall community level of ARG types.

The therapeutic dose of chlortetracycline caused the loss of Proteobacteria (from 83 to 75\%), which was mainly contributed by the decrease of Escherichia/Shigella $(P=0.028)$. Human fecal microbiome yet did not resemble to the pre-treatment status, even more than 1 year after antibiotic treatment [51]. The extent of recovery to the pre-treatment levels depends on diverse factors including microbiota structure, nature of antibiotic, course of treatment, and host genetics [52]. In the human study, two individuals responded to ciprofloxacin with a similar decrease of Proteobacteria, but these were distinct from variations of Actinobacteria, Bacteroidetes, Firmicutes, and Verrucomicrobia [53]. Alterations in fecal taxonomic phyla depend on class and number of courses of antibiotics [54], as well as diet [55-57], age [58], geographic location [59], and disease status [60].

Tracking the dynamics of ARG-harboring bacterial hosts under antibiotic exposure at the community level is central to understanding the emergence of resistance in microbial ecosystems. We determined the distribution of ARG-harboring bacterial hosts at the taxonomic genus level and tracked the effects of chlortetracycline treatment. The ARG-harboring genera at the community-wide level indicated that the fecal resistome resided in phylogenetically diverse microbiota such as Escherichia, Klebsiella, Shigella, and Salmonella. We also found that same ARG type was present in different genera but had preferences. For example, more than half of the genes resistant to multidrug (85\%), tetracycline (95\%), aminoglycoside (80\%), macrolide lincosamide streptogramin (76\%), and $\beta$-lactam (79\%) resided in Escherichia while vancomycin resistance 
genes mainly distributed in Enterococcus (100\%). These results supplement our understanding of the distribution of ARGs and their bacterial hosts in animal fecal metagenomes and provide precise administration strategy to treat resistant pathogens. For example, Enterococcus was the predominant bacterial host resistant to vancomycin, so we should treat Enterococcus infection using the effective antimicrobials that Enterococcus was sensitive to. We provide a novel insight into precision medicine through specific targeted administration of antimicrobials against resistant bacteria.

Antibiotic-alteration of bacterial community structure significantly influenced ARG-harboring bacterial hosts. We found that inhibition of Escherichia by a therapeutic dose of chlortetracycline as revealed by the bacterial community structure was the primary reason for the decrease of multidrug resistance genes. In addition, the enrichment of Bifidobacterium imposed by a therapeutic dose of chlortetracycline was due to the tetracycline resistance gene tet $\mathrm{W}$ they harbored. The antibacterial activity of chlortetracycline selected for resistant bacteria and inhibited the sensitive bacteria. This caused shifts in bacterial abundances that in turn changed resistome structure in the fecal metagenome. The concomitant ARG alterations and their bacterial hosts suggested the presence of widespread association between the fecal resistome and the bacterial community structure. Our findings suggested that antibiotic-mediated alteration of bacterial community structure shapes ARG contents in fecal microbiota of chickens under chlortetracycline treatment.

\section{Conclusions}

We conducted an integrated analysis of changes in microbiome structure and variations of ARG contents to obtain a comprehensive view of the fecal ecosystem dynamics following antibiotic treatment. The effects of chlortetracycline on the fecal resistome were dependent on the specific ARG subtypes and not simply overall ARGs in the community level. The changes in the structure of the antibiotic-induced fecal microbial community accompany changes in the abundance of bacterial hosts carrying specific ARGs. When the conclusions were adapted in other studies, many factors should be considered, including antibiotic spectrum, duration, and delivery route, as well as antibiotic susceptibility, microbial community structure, and contig assembly.

\section{Additional files}

Additional file 1: Figure S1. Animal groups and sampling time points. Figure S2. ARG types in chicken fecal metagenomes. Figure S3.

Comparisons of the genetic environment of $\mathrm{mcr}-1$. Figure S4. Changes in taxonomic phyla over time in different samples. Figure S5. Principal

component analysis of taxonomic genera in the therapeutic dose group and in the control group. Figure S6. ARGs and their bacterial hosts. Figure S7. Changes in dominated bacterial hosts carrying ARGs in the therapeutic group. (DOCX $1230 \mathrm{~kb}$ )

Additional file 2: Table S1. Information on sequencing depth of $16 \mathrm{~S}$ rRNA genes. Table S2. Information on assembly, contigs, and ORFs. Table S3. Relative abundance of ARG types. Table S4. Relative abundance of ARG subtypes and their significance in different groups. Table S5. Changes in taxonomic phyla over time in different samples (\%). Table S6. Changes in taxonomic genera over time in the therapeutic dose group and in the control group (\%). Table S7. Average percentages of bacterial hosts carrying antibiotic resistance genes (\%). Table S8. Variations of predominant ARGs carried by bacterial hosts over time in the therapeutic group. Table S9. Details of bacterial hosts and their harboring ARGs in the control group and in the therapeutic dose group on T5. Table S10. Changes in predominant bacterial hosts carrying ARGs in the therapeutic group. (XLSX $120 \mathrm{~kb}$ )

\section{Abbreviations}

ARGs: Antibiotic resistance genes; HGT: Horizontal gene transfer;

OTU: Operational taxonomic units

\section{Acknowledgements}

The authors are grateful to the anonymous reviewers for their insightful suggestions and comments.

\section{Funding}

This work was supported in part by the National Natural Science Foundation of China (no. 31702290) and the GRF7201/11E of Hong Kong and the National Natural Science Foundation of China (no. 31672608).

\section{Availability of data and materials}

The datasets supporting the conclusions of this article are available in the NCBI Sequence Read Archive (SRA) repository (accession no. SRP091637 for metagenomic analysis and accession no. SRP091694 for 16 S analysis).

\section{Authors' contributions}

WGX analyzed the data and wrote the manuscript. YLW, YXS, LPM, XTJ, and ADL analyzed the data and prepared figures. QLZ collected samples. ZLZ and TZ conceived the study. All authors edited the manuscript and approved the final draft.

\section{Ethics approval and consent to participate}

The study was approved by the Animal Experimental Ethics Committee of the South China Agricultural University (approval number 2015-013).

\section{Consent for publication}

Not applicable.

\section{Competing interests}

The authors declare that they have no competing interests.

\section{Publisher's Note}

Springer Nature remains neutral with regard to jurisdictional claims in published maps and institutional affiliations.

Received: 14 July 2017 Accepted: 30 January 2018

Published online: 13 February 2018

References

1. Kumarasamy KK, Toleman MA, Walsh TR, Bagaria J, Butt F, Balakrishnan R, et al. Emergence of a new antibiotic resistance mechanism in India, Pakistan, and the UK: a molecular, biological, and epidemiological study. Lancet Infect Dis. 2010;10(9):597-602.

2. Munoz-Price LS, Poirel L, Bonomo RA, Schwaber MJ, Daikos GL, Cormican M, et al. Clinical epidemiology of the global expansion of Klebsiella pneumoniae carbapenemases. Lancet Infect Dis. 2013;13(9):785-96.

3. Liu Y-Y, Wang Y, Walsh TR, Yi L-X, Zhang R, Spencer J, et al. Emergence of plasmid-mediated colistin resistance mechanism MCR-1 in animals and 
human beings in China: a microbiological and molecular biological study. Lancet Infect Dis. 2016;16(2):161-8.

4. Wang Y, Zhang R, Li J, Wu Z, Yin W, Schwarz S, et al. Comprehensive resistome analysis reveals the prevalence of NDM and MCR-1 in Chinese poultry production. Nat Microbiol. 2017;2:16260.

5. Allen HK. Antibiotic resistance gene discovery in food-producing animals. Curr Opin Microbiol. 2014;19:25-9.

6. McEachran AD, Blackwell BR, Hanson JD, Wooten K, Mayer GD, Cox SB, et al. Antibiotics, bacteria, and antibiotic resistance genes: aerial transport from cattle feed yards via particulate matter. Environ Health Perspect (Online). 2015;123(4):337.

7. Udikovic-Kolic N, Wichmann F, Broderick NA, Handelsman J. Bloom of resident antibiotic-resistant bacteria in soil following manure fertilization. Proc Natl Acad Sci. 2014;111(42):15202-7.

8. Xiong W, Wang M, Dai J, Sun Y, Zeng Z. Application of manure containing tetracyclines slowed down the dissipation of tet resistance genes and caused changes in the composition of soil bacteria. Ecotoxicol Environ Saf. 2018;147:455-60

9. DESA U. World population projected to reach 9.7 billion by 2050. 2015 .

10. National Bureau of statistics of China. http:/www.stats.gov.cn/english/. Accessed 17 Nov 2015

11. Van Boeckel TP, Brower C, Gilbert M, Grenfell BT, Levin SA, Robinson TP, et al. Global trends in antimicrobial use in food animals. Proc Natl Acad Sci. 2015;112(18):5649-54.

12. Oakley BB, Buhr RJ, Ritz CW, Kiepper BH, Berrang ME, Seal BS, et al. Successional changes in the chicken cecal microbiome during 42 days of growth are independent of organic acid feed additives. BMC Vet Res. 2014;10(1):282.

13. Costa MC, Bessegatto JA, Alfieri AA, Weese JS, João Filho A, Oba A. Different antibiotic growth promoters induce specific changes in the cecal microbiota membership of broiler chicken. PLoS One. 2017;12(2):e0171642.

14. Wei S, Gutek A, Lilburn M, Yu Z. Abundance of pathogens in the gut and litter of broiler chickens as affected by bacitracin and litter management. Vet Microbiol. 2013;166(3):595-601.

15. Fang H, Han L, Cui Y, Xue Y, Cai L, Yu Y. Changes in soil microbial community structure and function associated with degradation and resistance of carbendazim and chlortetracycline during repeated treatments. Sci Total Environ. 2016;572:1203-12

16. QYResearch Medical Research Centre. 2015 Market research report on global and China feed chlortetracycline industry. http://www.qyresearch. com (accessed Oct 10, 2017)

17. Lekunberri I, Subirats J, Borrego CM, Balcázar JL. Exploring the contribution of bacteriophages to antibiotic resistance. Environ Pollut. 2017;220:981-4.

18. Enault F, Briet A, Bouteille L, Roux S, Sullivan MB, Petit M-A. Phages rarely encode antibiotic resistance genes: a cautionary tale for virome analyses. ISME J. 2017;11(1):237.

19. Forsberg K, Patel S, Gibson MK, Lauber CL, Knight R, Fierer N, et al. Bacterial phylogeny structures soil resistomes across habitats. Nature. 2014;509(7502):612.

20. Jia S, Shi P, Hu Q, Li B, Zhang T, Zhang X-X. Bacterial community shift drives antibiotic resistance promotion during drinking water chlorination. Environ Sci Technol. 2015;49(20):12271-9.

21. Fang $\mathrm{H}$, Wang H, Cai L, Yu Y. Prevalence of antibiotic resistance genes and bacterial pathogens in long-term manured greenhouse soils as revealed by metagenomic survey. Environ Sci Technol. 2015;49(2):1095-104.

22. Sommer MO, Church GM, Dantas G. The human microbiome harbors a diverse reservoir of antibiotic resistance genes. Virulence. 2010;1(4):299-303.

23. Gibson MK, Forsberg KJ, Dantas G. Improved annotation of antibiotic resistance determinants reveals microbial resistomes cluster by ecology. ISME J. 2015;9(1):207-16.

24. Stanley D, Geier MS, Hughes RJ, Denman SE, Moore RJ. Highly variable microbiota development in the chicken gastrointestinal tract. PLoS One. 2013;8(12):e84290.

25. Ranjitkar S, Lawley B, Tannock G, Engberg RM. Bacterial succession in the broiler gastrointestinal tract. Appl Environ Microbiol. 2016;82(8):2399-410.

26. Zhou W, Wang Y, Lin J. Functional cloning and characterization of antibiotic resistance genes from the chicken gut microbiome. Appl Environ Microbiol. 2012;78(8):3028-32.

27. Ma L, Xia Y, Li B, Yang Y, Li L-G, Tiedje JM, et al. Metagenomic assembly reveals hosts of antibiotic resistance genes and the shared resistome in pig chicken, and human feces. Environ Sci Technol. 2015;50(1):420-7.
28. Li B, Yang Y, Ma L, Ju F, Guo F, Tiedje JM, et al. Metagenomic and network analysis reveal wide distribution and co-occurrence of environmental antibiotic resistance genes. ISME J. 2015;9(11):2490-502.

29. Allen HK, Stanton TB. Altered egos: antibiotic effects on food animal microbiomes. Annu Rev Microbiol. 2014;68:297-315.

30. Fadrosh DW, Ma B, Gajer P, Sengamalay N, Ott S, Brotman RM, et al. An improved dual-indexing approach for multiplexed 165 rRNA gene sequencing on the Illumina MiSeq platform. Microbiome. 2014;2(1):1.

31. Kozich JJ, Westcott SL, Baxter NT, Highlander SK, Schloss PD. Development of a dual-index sequencing strategy and curation pipeline for analyzing amplicon sequence data on the MiSeq Illumina sequencing platform. Appl Environ Microbiol. 2013;79(17):5112-20.

32. Caporaso JG, Kuczynski J, Stombaugh J, Bittinger K, Bushman FD, Costello EK, et al. QIIME allows analysis of high-throughput community sequencing data. Nat Methods. 2010;7(5):335-6.

33. Cole JR, Wang Q, Fish JA, Chai B, McGarrell DM, Sun Y, et al. Ribosomal Database Project: data and tools for high throughput rRNA analysis. Nucleic Acids Res. 2013;42(D1):D633-D42.

34. Yan L, Yang M, Guo H, Yang L, Wu J, Li R, et al. Single-cell RNA-Seq profiling of human preimplantation embryos and embryonic stem cells. Nat Struct Mol Biol. 2013;20(9):1131-9.

35. Yang $Y$, Jiang $X$, Chai B, Ma L, Li B, Zhang A, et al. ARGs-OAP: online analysis pipeline for antibiotic resistance genes detection from metagenomic data using an integrated structured ARG-database. Bioinformatics. 2016;32(15): 2346-51.

36. Yang Y, Li B, Ju F, Zhang T. Exploring variation of antibiotic resistance genes in activated sludge over a four-year period through a metagenomic approach. Environ Sci Technol. 2013;47(18):10197-205.

37. Hyatt D, Chen G-L, LoCascio PF, Land ML, Larimer FW, Hauser LJ. Prodigal: prokaryotic gene recognition and translation initiation site identification. BMC bioinformatics. 2010;11(1):1.

38. Hu Y, Yang X, Qin J, Lu N, Cheng G, Wu N, et al. Metagenome-wide analysis of antibiotic resistance genes in a large cohort of human gut microbiota. Nat commun. 2013:4:2151.

39. Huson DH, Mitra S, Ruscheweyh H-J, Weber N, Schuster SC. Integrative analysis of environmental sequences using MEGAN4. Genome Res. 2011; 21(9):1552-60

40. Ishii SI, Suzuki S, Norden-Krichmar TM, Tenney A, Chain PS, Scholz MB, et al. A novel metatranscriptomic approach to identify gene expression dynamics during extracellular electron transfer. Nat Commun. 2013;4:1601.

41. Eckburg PB, Bik EM, Bernstein CN, Purdom E, Dethlefsen L, Sargent M, et al. Diversity of the human intestinal microbial flora. Science. 2005; 308(5728):1635-8

42. Tang Y, Underwood A, Gielbert A, Woodward MJ, Petrovska L. Metaproteomics analysis reveals the adaptation process for the chicken gut microbiota. Appl Environ Microbiol. 2014;80(2):478-85.

43. Hou Q, Kwok L-Y, Zheng Y, Wang L, Guo Z, Zhang J, et al. Differential fecal microbiota are retained in broiler chicken lines divergently selected for fatness traits. Sci Rep. 2016:6:37376.

44. Wichmann F, Udikovic-Kolic N, Andrew S, Handelsman J. Diverse antibiotic resistance genes in dairy cow manure. MBio. 2014;5(2):e01017-3.

45. Wang $Y$, Tian G-B, Zhang R, Shen Y, Tyrrell JM, Huang X, et al. Prevalence, risk factors, outcomes, and molecular epidemiology of mcr-1-positive Enterobacteriaceae in patients and healthy adults from China: an epidemiological and clinical study. Lancet Infect Dis. 2017;17(4):390-9.

46. Crofts TS, Gasparrini AJ, Dantas G. Next-generation approaches to understand and combat the antibiotic resistome. Nat Rev Microbiol. 2017; 15(7):422.

47. Zhu Y-G, Johnson TA, Su J-O, Oiao M, Guo G-X, Stedtfeld RD, et al. Diverse and abundant antibiotic resistance genes in Chinese swine farms. Proc Natl Acad Sci. 2013;110(9):3435-40.

48. Forsberg KJ, Reyes A, Wang B, Selleck EM, Sommer MO, Dantas G. The shared antibiotic resistome of soil bacteria and human pathogens. Science. 2012;337(6098):1107-11.

49. Moore AM, Ahmadi S, Patel S, Gibson MK, Wang B, Ndao IM, et al. Gut resistome development in healthy twin pairs in the first year of life. Microbiome. 2015;3(1):1.

50. Rampelli S, Schnorr SL, Consolandi C, Turroni S, Severgnini M, Peano C, et al. Metagenome sequencing of the Hadza hunter-gatherer gut microbiota. Curr Biol. 2015:25(13):1682-93. 
51. Voigt AY, Costea PI, Kultima JR, Li SS, Zeller G, Sunagawa S, et al. Temporal and technical variability of human gut metagenomes. Genome Biol. 2015;16(1):1.

52. Schulfer A, Blaser MJ. Risks of antibiotic exposures early in life on the developing microbiome. PLoS Pathog. 2015;11(7):e1004903.

53. Willmann M, El-Hadidi M, Huson DH, Schütz M, Weidenmaier C, Autenrieth $\mathrm{IB}$, et al. Antibiotic selection pressure determination through sequencebased metagenomics. Antimicrob Agents Chemother. 2015;59(12):7335-45.

54. Nobel YR, Cox LM, Kirigin FF, Bokulich NA, Yamanishi S, Teitler I, et al. Metabolic and metagenomic outcomes from early-life pulsed antibiotic treatment. Nat commun. 2015;6:7486.

55. Flint HJ, Duncan SH, Scott KP, Louis P. Links between diet, gut microbiota composition and gut metabolism. Proc Nutr Soc. 2015;74(01):13-22.

56. Carmody RN, Gerber GK, Luevano JM, Gatti DM, Somes L, Svenson KL, et al. Diet dominates host genotype in shaping the murine gut microbiota. Cell Host Microbe. 2015;17(1):72-84

57. David LA, Maurice CF, Carmody RN, Gootenberg DB, Button JE, Wolfe BE, et al. Diet rapidly and reproducibly alters the human gut microbiome. Nature. 2014:505(7484):559-63.

58. OToole PW, Jeffery IB. Gut microbiota and aging. Science. 2015;350(6265):1214-5.

59. Yatsunenko T, Rey FE, Manary MJ, Trehan I, Dominguez-Bello MG, Contreras $\mathrm{M}$, et al. Human gut microbiome viewed across age and geography. Nature. 2012;486(7402):222-7.

60. Kostic AD, Gevers D, Siljander H, Vatanen T, Hyötyläinen T, Hämäläinen $A-M$, et al. The dynamics of the human infant gut microbiome in development and in progression toward type 1 diabetes. Cell Host Microbe. 2015;17(2):260-73.

\section{Submit your next manuscript to BioMed Central and we will help you at every step:}

- We accept pre-submission inquiries

- Our selector tool helps you to find the most relevant journal

- We provide round the clock customer support

- Convenient online submission

- Thorough peer review

- Inclusion in PubMed and all major indexing services

- Maximum visibility for your research

Submit your manuscript at www.biomedcentral.com/submit

C) Biomed Central 